\title{
Green and efficient synthesis of some pyrido [2,3-d]pyrimidin-4(3h)-one derivatives via iodine catalyst in aqueous media and evaluation the synthesized compounds as anticancer
}

\author{
Ayman M. F. Elgohary ${ }^{1,2, *}$, E. M. Ezz El-Arab ${ }^{2}$ \\ ${ }^{1}$ Department of medical laboratories College of Applied Medical Science, Majmaah University, Almajma'ah 11952, KSA \\ ${ }^{2}$ National Organization For Drug Control \& Research, Pharmaceutical Chemistry Division, Giza, Egypt
}

Email address:

Elgohary431974@yahoo.com (A. M. F. Elgohary)

\section{To cite this article:}

Ayman M. F. Elgohary, E. M. Ezz El-Arab. Green and Efficient Synthesis of Some Pyrido[2,3-D]Pyrimidin-4(3h)-One Derivatives Via Iodine Catalyst in Aqueous Media and Evaluation the Synthesized Compounds as Anticancer, Science Journal of Chemistry. Vol. 1, No. 1, 2013, pp. 1-6. doi: 10.11648/j.sjc.20130101.11

\begin{abstract}
A series of new 2-propylpyrido[2,3-d]pyrimidin-4(3H)-one with different substituents at position 3 were synthesized by traditional method and Iodine catalyst method. The effect of the newly synthesized compounds was tested as anti-cancer in National Cancer Institute(NCI)in USA. Some of the synthesized compounds exploited potent antitumor activity, especially the compounds pyrido[2,3-d][1,3] oxazin (2), 3-amino derivative $3 \mathrm{a}, 3 \mathrm{~b}$ and hydroxy derivative $3 \mathrm{c}$ displayed the highest activity among the test compounds with IC50 $<5 \mathrm{mg} / \mathrm{mL}$
\end{abstract}

Keywords: Pyrido[2,3-D][1,3]Oxazin-4-One, 2-Propylpyrido[2,3-D]Pyrimidin-4(3h)-One, Antitumor

\section{Introduction}

Cancer is the second leading cause of death in the world. The pyrido[2,3-d]pyrimidine nucleus represents in many biologically active compounds which includes antitumour [1-7], antibacterial [8], anticonvulsant [9], antipyretic [10], analgesic [11], and CNS depressant activity [12]. Specifically pyrido[2,3-d]pyrimidines known to inhibit Pneumocystis carinii(pc), Toxoplasma gondii(tg) of tumor cell lines in culture [13] and the activity is attributed to inhibition of dihydrofolate reductase (DHFR) [14,15]. In modern synthetic organic chemistry, development of a novel and efficient method for synthesis of pyridopyrimidine and its biological activity is one of the current areas of research interest. In this work, we aimed to synthesize new 2propylpyrido[2,3-d]pyrimidin-4(3H)-one derivatives bearing different substituents at position $3\left[\mathrm{NH}_{2}, \mathrm{~N}\right.$-aryl] in order to, examine the effect of substitution at position 3 on the antitumor activity.

\section{Result and Discussion}

2-propyl- 4H- pyrido [2,3-d] [1,3] oxazin- 4- one (2) has been synthesized from the interaction of butyroyl chloride with 2-aminonicotinic acid in pyridine to give 2-propyl4H-pyrido[2,3-d][1,3] oxazin-4-one (2). The structure of compound 2 was inferred from its IR spectra $\left(\mathrm{cm}^{-1}\right)$ which exhibits strong absorption bands at 1614, $1764\left(\mathrm{~cm}^{-1}\right)$ due to $v_{\max }$ of $\mathrm{C}=\mathrm{N}$ and $\mathrm{C}=\mathrm{O}$ of pyridoxazinone and lack of any band for $\mathrm{NH}$ and / or $\mathrm{OH}$. The starting compound $3 \mathrm{a}$ was prepared via reacting pyridooxazinone (2) with hydrazine hydrate in ethanol in water path, the formation of compound $3 \mathrm{a}$ was confirmed by ${ }^{1} \mathrm{HNMR}$ that showed singlet signal at $\square$ 9.8ppm and from its IR spectrum which exhibits strong absorption bands at 1614 and $1670\left(\mathrm{~cm}^{-1}\right)$ due to $v_{\max }$ of $\mathrm{C}=\mathrm{N}, \mathrm{C}=\mathrm{O}$ and lack of any band for of $\mathrm{C}=\mathrm{O}$ of pyridoxazinone, also compound 2 was reacted with ammonium acetate in an oil bath to form 2-propylpyrido[2,3d]pyrimidin-4(3H)-one (3b). Beside the same compound $3 \mathrm{~b}$ was obtained by heating compound 2 in formamide under reflux. On a reaction of compound 2 with hydroxyl amine gave 3-hydroxy-2-propylpyrido[2,3-d]pyrimidin$4(3 \mathrm{H})$-one $(3 \mathrm{c})$.

On the other hand compounds $3 \mathrm{~d}-\mathrm{f}$ were prepared by interaction of compound 2 with aromatic amines namely orth, meta and para toluidine. The structure of compounds $3 \mathrm{~d}-\mathrm{f}$ were inferred from its IR spectrum which exhibits strong absorption bands at 1614 and $1670\left(\mathrm{~cm}^{-1}\right)$ due to $v_{\max }$. of 
$\mathrm{C}=\mathrm{N}, \mathrm{C}=\mathrm{O}$ and lack of any band for of $\mathrm{C}=\mathrm{O}$ of pyridoxazinone.

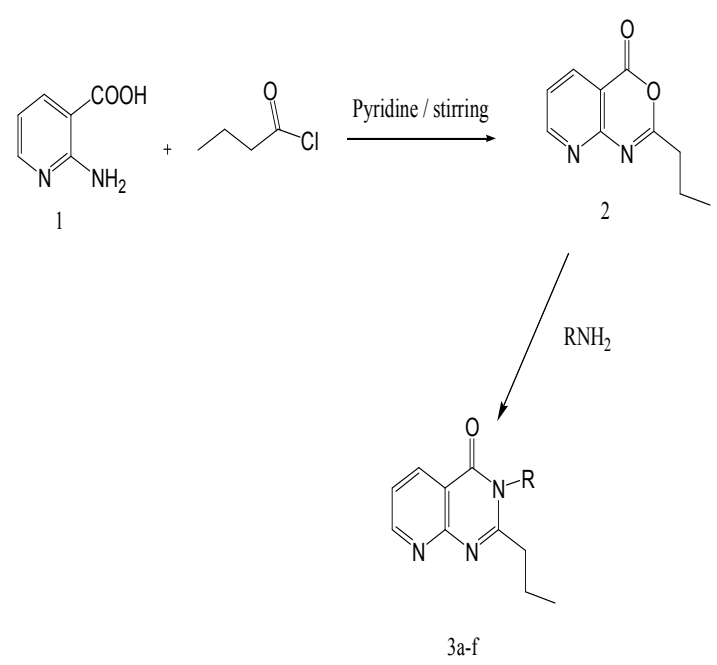

Scheme 1

Table 1. Synthesis of 2-propylpyrido[2,3-d]pyrimidin-4(3H)-one and its derivatives $3 a-c$.

\begin{tabular}{|c|c|c|c|c|c|}
\hline $\begin{array}{l}\text { Comb. } \\
\text { No. }\end{array}$ & $\mathbf{R}$ & $\begin{array}{l}\text { Molecular } \\
\text { formula } \\
\text { M.wt }\end{array}$ & $\begin{array}{l}\text { Reagent and } \\
\text { condition }\end{array}$ & $\begin{array}{l}\text { Time } \\
\text { (h) }\end{array}$ & $\mathrm{mp}\left({ }^{\circ} \mathrm{C}\right)$ \\
\hline $3 a$ & $\mathrm{NH}_{2}$ & $\begin{array}{l}\mathrm{C}_{10} \mathrm{H}_{12} \mathrm{~N}_{4} \mathrm{O} \\
204\end{array}$ & $\mathrm{NH}_{2} \mathrm{NH}_{2} / \mathrm{EtOH}$ & 0.5 & 130 \\
\hline \multirow{2}{*}{$3 b$} & $\mathrm{H}$ & $\begin{array}{l}\mathrm{C}_{10} \mathrm{H}_{11} \mathrm{~N}_{3} \mathrm{O} \\
189\end{array}$ & formamide & 3 & 286 \\
\hline & $\mathrm{H}$ & $\begin{array}{l}\mathrm{C}_{10} \mathrm{H}_{11} \mathrm{~N}_{3} \mathrm{O} \\
189\end{array}$ & \multicolumn{2}{|l|}{$\begin{array}{l}\mathrm{CH}_{3} \mathrm{COONH}_{4} \mathrm{oil}_{3} \\
\text { bath } / 150{ }^{\circ} \mathrm{C}\end{array}$} & 286 \\
\hline $3 c$ & $\mathrm{OH}$ & $\begin{array}{l}\mathrm{C}_{10} \mathrm{H}_{11} \mathrm{~N}_{3} \mathrm{O}_{2} \\
205\end{array}$ & $\begin{array}{l}\mathrm{NH}_{2} \mathrm{OH} / \quad \mathrm{EtOH} \\
\text { reflux }\end{array}$ & $\mathrm{H}_{3}$ & 165 \\
\hline $3 d$ & & $\begin{array}{l}\mathrm{C}_{17} \mathrm{H}_{17} \mathrm{~N}_{3} \mathrm{O} \\
279\end{array}$ & EtOH reflux & 4.5 & 234 \\
\hline $3 e$ & & $\begin{array}{l}\mathrm{C}_{17} \mathrm{H}_{17} \mathrm{~N}_{3} \mathrm{O} \\
279\end{array}$ & EtOH reflux & 5 & 255 \\
\hline $3 \mathrm{f}$ & & $\begin{array}{l}\mathrm{C}_{17} \mathrm{H}_{17} \mathrm{~N}_{3} \mathrm{O} \\
279\end{array}$ & EtOH reflux & 5 & 195 \\
\hline
\end{tabular}

On the other hand Three methods were investigated for the synthesis of 3-(Arylylideneamino)-2- propylpyrido [2,3-d]pyrimidin-4(3H)-ones $4 \mathrm{a}-\mathrm{k}$, the first is method A (traditional method) by condensation of 3- amino- 2- propylpyrido[2,3-d] pyrimidin-4(3H)-one (3b) and aryl aldehydes in refluxing ethanol but this methods suffering from high temperature, low yield, long reaction time. As part of our interests in the synthesis of pyridopyrimidine and due to the present awareness of applying environmentally benign strategies in organic synthesis, in this communication we report the $\mathrm{I} 2 / \mathrm{KI}$ mediated condensation of 3amino- 2- propylpyrido [2,3-d] pyrimidin- $4(3 \mathrm{H})$-one (3a) with various aldehydes for preparation of 3 (Arylylideneamino) - 2- propylpyrido [2,3-d] pyrimidin $4(3 \mathrm{H})$ - ones $4 \mathrm{a}-\mathrm{k}$ in ethanol-water (method $\mathrm{B}$ ) or boiling water (method C).

Initially, a solution of $\mathrm{I}_{2} / \mathrm{KI}$ was prepared by dissolving specified molecular iodine in a saturated aqueous solution of potassium iodide. We were delighted to find that expo sure of 3-amino-2-propylpyrido [2,3-d] pyrimidin - 4(3H) one (3a) with aromatic aldehydes on stirring or refluxing in equimolar aqueous solution of $\mathrm{I}_{2} / \mathrm{KI}$ offered the 3 (Arylylideneamino) - 2- propylpyrido [2,3-d] pyrimidin$4(3 \mathrm{H})$-ones $4 \mathrm{a}-\mathrm{k}$ in good to excellent isolated yields (Scheme 2).

To assess the catalytic effect of $\mathrm{I}_{2} / \mathrm{KI}$ system, the typical reaction of 3- amino -2- propylpyrido [2,3-d] pyrimidin$4(3 \mathrm{H})$-one (3a) with benzaldehyde was attempted in the absence of $\mathrm{I}_{2} / \mathrm{KI}$ catalyst at room temperature and also at reflux temperature, but only the starting materials were recovered. It can be assumed that in the $\mathrm{I}_{2} / \mathrm{KI}$ catalytic system, molecular iodine acts as a mild Lewis acid and oxidant and $\mathrm{KI}$ as a solubilizing agent of molecular iodine in water. On the other hand water as the reaction media is mostly suitable because the products are practically insoluble in water and the work-up process can be much simplified. The reaction was performed in two methods. In method (B), reactants were first dissolved in minimum ethanol (ca. $1 \mathrm{~mL}$ ) in order to make the mixture homogeneous then, the $I_{2} / K I$ aqueous solution was added and stirred at room temperature for desired times indicated in Table 2. Progress of the reaction was monitored by TLC. In method $(\mathrm{C})$, the reaction was only conducted in $\mathrm{I}_{2} / \mathrm{KI}$ aqueous solution at reflux conditions. The products from both procedures were isolated in a practically pure form by simple Buchner filtration of the final aqueous mixture. The structures of these compounds were established by their physical and spectral data (Table 2).

From the data in table (2) it can be concluded that the presence of the electron-withdrawing substituents on the aromatic ring like entry $4 \mathrm{c}$ and $4 \mathrm{~d}$ can increase the reaction rate and as the result the reaction times are shortened and the yields are increased while the electron donating substituents have the diverse effect. It should be pointed out here that yields from method (B) are higher compared with that of method (C) and yields from method (C) are higher compared with that of method (A). But the reactions in method (C) showed an attractive feature from the viewpoint of environmental benign. 
Table 2. Synthesis of 3-(Arylylideneamino)-2-propylpyrido[2,3-d]pyrimidin-4(3H)-ones 4a-k.

\begin{tabular}{|c|c|c|c|c|c|c|c|c|c|}
\hline \multirow[t]{2}{*}{ Entry } & \multirow[t]{2}{*}{ Ar } & \multirow[t]{2}{*}{ Molecular formula } & \multicolumn{2}{|c|}{$\begin{array}{l}\text { Method A } \\
\text { Traditional method }\end{array}$} & \multicolumn{2}{|c|}{$\begin{array}{l}\text { Method B } \\
\mathbf{I}_{2} / \mathbf{K I} \\
\text { in Ethanol/ water }\end{array}$} & \multicolumn{2}{|l|}{$\begin{array}{l}\text { Method C } \\
\mathbf{I}_{2} / \mathbf{K I} \\
\text { in Water }\end{array}$} & \multirow{2}{*}{$\mathbf{m p}\left({ }^{\circ} \mathrm{C}\right)$} \\
\hline & & & Yield \% & Time (h) & Yield \% & Time (min) & Yield \% & Time(min) & \\
\hline $4 a$ & & $\begin{array}{l}\mathrm{C}_{17} \mathrm{H}_{16} \mathrm{~N}_{4} \mathrm{O} \\
292\end{array}$ & 80 & 4 & 91 & 25 & 85 & 40 & 233 \\
\hline $4 b$ & & $\begin{array}{l}\mathrm{C}_{18} \mathrm{H}_{18} \mathrm{~N}_{4} \mathrm{O} \\
308\end{array}$ & 73 & 6 & 93 & 35 & 82 & 45 & 238 \\
\hline $4 c$ & & $\begin{array}{l}\mathrm{C}_{17} \mathrm{H}_{15} \mathrm{~N}_{4} \mathrm{OCl} \\
262.5\end{array}$ & 80 & 5 & 95 & 20 & 89 & 60 & 302 \\
\hline $4 d$ & & $\begin{array}{l}\mathrm{C}_{17} \mathrm{H}_{15} \mathrm{~N}_{5} \mathrm{O}_{3} \\
337\end{array}$ & 75 & 3.5 & 92 & 15 & 87 & 45 & 315 \\
\hline $4 \mathrm{e}$ & & $\begin{array}{l}\mathrm{C}_{17} \mathrm{H}_{15} \mathrm{~N}_{5} \mathrm{O}_{3} \\
337\end{array}$ & 60 & 4 & 95 & 30 & 83 & 50 & 305 \\
\hline $4 f$ & & $\begin{array}{l}\mathrm{C}_{18} \mathrm{H}_{18} \mathrm{~N}_{4} \mathrm{O}_{2} \\
322\end{array}$ & 74 & 5 & 83 & 45 & 78 & 60 & 216 \\
\hline $4 \mathrm{~g}$ & & $\begin{array}{l}\mathrm{C}_{18} \mathrm{H}_{18} \mathrm{~N}_{4} \mathrm{O}_{2} \\
322\end{array}$ & 77 & 7 & 94 & 30 & 80 & 60 & 226 \\
\hline $4 \mathrm{~h}$ & & $\begin{array}{l}\mathrm{C}_{18} \mathrm{H}_{18} \mathrm{~N}_{4} \mathrm{O}_{2} \\
322\end{array}$ & 82 & 6 & 91 & 30 & 84 & 90 & 219 \\
\hline $4 i$ & & $\begin{array}{l}\mathrm{C}_{17} \mathrm{H}_{16} \mathrm{~N}_{4} \mathrm{O}_{2} \\
310\end{array}$ & 75 & 5 & 97 & 30 & 82 & 120 & 254 \\
\hline $4 j$ & & $\begin{array}{l}\mathrm{C}_{17} \mathrm{H}_{16} \mathrm{~N}_{4} \mathrm{O}_{2} \\
310\end{array}$ & 70 & 5 & 87 & 35 & 80 & 120 & 266 \\
\hline $4 \mathrm{k}$ & & $\begin{array}{l}\mathrm{C}_{17} \mathrm{H}_{16} \mathrm{~N}_{4} \mathrm{O}_{2} \\
310 \\
\end{array}$ & 72 & 6 & 90 & 30 & 83 & 90 & 245 \\
\hline
\end{tabular}

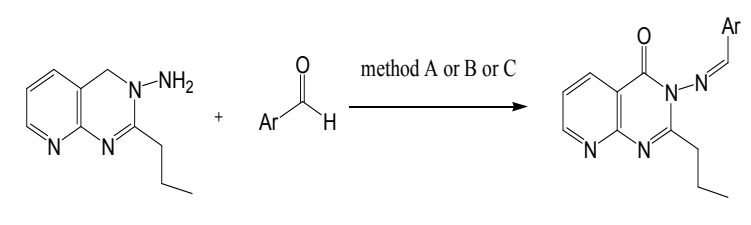

3a

$4 a-k$

Scheme 2

\section{Biological Part}

The Development Therapeutics Program (DTP), Division of Cancer Treatment and Diagnosis (DCTD), National Cancer Institute (NCI) USA has used an In-vitro model consisting of 60 human tumor cell lines as the primary anticancer screen. An analysis of the data indicated that approximately $95 \%$ of the actives from the 60 -cell line screen could be identified using only three cell lines. For this reason, the DTP has begun using, as its primary anti-cancer assay, a 3-cell lines panel consisting of NCI-H 460(Lung), MCF7 (Breast), and SF-268 (CNS). The NCI protocol has been described previously briefly. Cell lines were inocu- lated onto a series of 96-well plates. Seeding densities varied depending upon growth characteristics. After a $24 \mathrm{~h}-$ drug - free incubation, test compounds were added routinely at five tenfold dilutions starting at maximum 10-4M. After incubation periods of $48 \mathrm{~h}$ or 6 days, cell growth or viability was assayed using the sulphorhodamine B procedure42.The results of antineoplastic evaluation of the tested compounds it is evident that the all compounds showed cytotoxic effects and high selectivity against breast adenocarcenoma (MCF7), Doxorubicin which is one of the most effective anticancer agents was used as the reference drug in this study. The relationship between surviving fraction and drug concentration was plotted to obtain the survival curve of breast cancer cell line (MCF7). The response parameter calculated was the IC50 value, which corresponds to the concentration required for $50 \%$ inhibition of cell viability. The IC50 of the synthesized compounds compared to the reference drug are shown in Table 4. From the results in Table (3), it was found that the best results were obtained by compounds pyrido[2,3-d][1,3]oxazin (2), 3amino derivative $3 \mathrm{a}, 3 \mathrm{~b}$ and hydroxy derivative $3 \mathrm{c}$. Regarding the chloro $4 \mathrm{c}$ and hydroxyl substituted $4 \mathrm{i}-\mathrm{k}$ give moderate activity. Compound $3 \mathrm{~b}$ was the most potent com- 
pound in this screening with IC50 equal to $3.82 \mathrm{mg} / \mathrm{mL}$.. Further studies needed to be done to explore the mechanism of action as well as the effect of substitution at other positions of the ring.

Table 3. Results of in vitro cytotoxic activity of the synthesized compounds on human breast adenocarcinoma cell line (MCF7).

\begin{tabular}{ll}
\hline Compound no. & IC50 in $\mathbf{~ g / m L}$ \\
\hline 2 & 4.21 \\
$3 \mathrm{a}$ & 4.93 \\
$3 \mathrm{~b}$ & 3.82 \\
$3 \mathrm{c}$ & 4.77 \\
$3 \mathrm{~d}$ & 14.58 \\
$3 \mathrm{e}$ & 33.98 \\
$3 \mathrm{f}$ & 29.22 \\
$4 \mathrm{a}$ & 17.46 \\
$4 \mathrm{~b}$ & 20.5 \\
$4 \mathrm{c}$ & 11.12 \\
$4 \mathrm{~d}$ & $>50$ \\
$4 \mathrm{e}$ & $>50$ \\
$4 \mathrm{f}$ & 15.6 \\
$4 \mathrm{~g}$ & 11.23 \\
$4 \mathrm{~h}$ & 10.29 \\
$4 \mathrm{i}$ & 12.46 \\
$4 \mathrm{j}$ & 9.33 \\
$4 \mathrm{k}$ & 11.65 \\
Doxorubicin & 3.11 \\
\hline
\end{tabular}

\section{Conclusion}

The objective of the present study was to synthesize new pyrido [2,3-d]pyrimidinones by different method bearing different substituents at position 3 and to examine the effect of substitution at position 3 on the cytotoxic activity. Some of these new compounds exhibited good antitumor activity against MCF7 when compared to doxorubicin as a reference drug. The best results were obtained by derivatives bearing $\mathrm{NH}_{2}, \mathrm{OH}$ or $\mathrm{NH}$ groups at position 3 . The 3amino derivative $3 \mathrm{a}$ was the most potent compound in this screening with IC50 equal to $3.82 \mathrm{mg} / \mathrm{mL}$.

\section{Experimental}

All melting point are uncorrected and determined by the open capillary method using Gallen Kamp melting point apparatus. Microanalysis were carried out by the Micro Analytical Unit at Cairo University. IR spectra ( $\mathrm{KBr}$ disk) were recorded on FT/IR-300E Jasco spectrophotometer. HNMR spectra were recorded in $\mathrm{CDCl} 3$ or DMSO-d6 solution on a Varian EM 390-90 MHz43,44. Mass spectrometry were recorded were recorded Shomadzu, GC - MS (QP $-1000 \mathrm{EX}$

2-propyl-4H-pyrido[2,3-d][1,3]oxazin-4-one (2)

A stirred solution of 2-aminonicotinic acid $13.8 \mathrm{~g}(0.1$ mol) in dry pyridine $(150 \mathrm{ml})$ was treated drop wise with nbutyroyl chloride $10.5 \mathrm{~g}(0.11 \mathrm{~mol})$ during $10 \mathrm{~min}$. the mixture was stirred at room temperature (3hours) and poured into a mixture of ice and hydrochloric acid gave crude 2propyl-4H-pyrido[2,3-d][1,3]oxazin-4-one The precipitated solid was filtered off, washed with cold water and recrys- tallization from petroleum ether $40-60 \mathrm{oC}$, giving 2propyl-4H-pyrido[2,3-d][1,3]oxazin-4-one (2) as a colorless crystals melting point $(63 \mathrm{oC})(2) \mathrm{IR}(\mathrm{KBr}) \mathrm{cm}-1: 3055$ (CH aromatic), 2965, 2935, 2875 (CH aliphatic), $1764(\mathrm{C}=\mathrm{O}), 1614(\mathrm{C}=\mathrm{N}), 1161 \quad(\mathrm{C}-\mathrm{O}) ; \mathrm{MS}: \mathrm{m} / \mathrm{z} 190$ [M+]; Anal. calcd. for $\mathrm{C} 10 \mathrm{H} 10 \mathrm{~N} 2 \mathrm{O} 2$ : C, 63.15; H, 5.26; N, 14.73; found: C, 63.11; H, 5.24; N, 14.70

3-amino-2-propylpyrido[2,3-d]pyrimidin-4(3H)-one (3a)

Compound (2) (1.89 g- 10mmol) and $5 \mathrm{ml}$ hydrazine hydrate were heated in water bath for $1 / 2$ hour and then added $50 \mathrm{ml}$ ethanol the mixture was refluxed, The completion of the reaction was monitored by TLC using chloroform: methanol $(8: 2)$ as eluent and the obtained solid was filtered off and recrystalization from petroleum ether $60-80{ }^{\circ} \mathrm{C}$ to give 3-amino-2-propylpyrido[2,3-d]pyrimidin-4(3H)-one (3a). $\operatorname{IR}(\mathrm{KBr}) \mathrm{cm}^{-1}: 1596(\mathrm{C}=\mathrm{N}), 1673(\mathrm{C}=\mathrm{O}), 2875,2935,2965$ (CH aliphatic), 3055 (CH aromatic), 3309, 3212( $\left.\mathrm{NH}_{2}\right)$; MS: $\mathrm{m} / \mathrm{z} 204\left[\mathrm{M}^{+}\right] ;{ }^{1} \mathrm{HNMR}\left(\mathrm{CHCl}_{3}\right): \delta 9.8\left(\mathrm{~s}, 2 \mathrm{H}, \mathrm{NH}_{2}\right), 7.6-$ $8.8\left(\mathrm{~m}, 3 \mathrm{H}\right.$, aromatic), $2.7\left(\mathrm{t}, 2 \mathrm{H}, \mathrm{CH}_{2}\right.$ pyrimidine $), 1.9$ (sextet,2H, $\mathrm{CH}_{2} \mathrm{Me}$ ), 1.1(t, 3H, $\left.\mathrm{CH}_{3}\right)$. Anal. calcd. for $\mathrm{C}_{10} \mathrm{H}_{12} \mathrm{~N}_{4} \mathrm{O}$ : C, 58.82; H, 5.88; N, 27.45; found: C, 58.80; $\mathrm{H}, 5.84 ; \mathrm{N}, 27.41$.

2-propylpyrido[2,3-d]pyrimidin-4(3H)-one (3b)

method A- 2-propyl-4H-pyrido[2,3-d][1,3]oxazin-4-one (2) $(1 \mathrm{mmol})$ was heated with ammonium acetate $(4 \mathrm{mmol})$ in an oil bath at $150{ }^{\circ} \mathrm{C}$ for two hours, the reaction mixture was cooled and poured in cold water. The precipitated solid was filtered off, washed with water and recrystallization from ethanol to give 3b. IR(KBr) cm $\mathrm{cm}^{-1}: 3215(\mathrm{NH}), 3024$ (CH aromatic), 2969, 2935, 2905, 2877 (CH aliphatic), $1675(\mathrm{C}=\mathrm{O}), 1597(\mathrm{C}=\mathrm{N})$; MS: m/z $189\left[\mathrm{M}^{+}\right]$; ${ }^{1} \mathrm{HNMR}$ (DMSO) : $\delta 8.9(\mathrm{~s}, \mathrm{H}, \mathrm{NH}), 7.2-8.2(\mathrm{~m}, 3 \mathrm{H}$, aromatic), 1.1(t, $\left.3 \mathrm{H}, \mathrm{CH}_{3}\right), 1.9$ (sextet, $\left.2 \mathrm{H}, \mathrm{CH}_{2} \mathrm{Me}\right), 2.7\left(\mathrm{t}, 2 \mathrm{H}, \mathrm{CH}_{2}\right)$; Anal. calcd. for $\mathrm{C}_{10} \mathrm{H}_{11} \mathrm{~N}_{3} \mathrm{O}: \mathrm{C}, 63.49 ; \mathrm{H}, 5.82 ; \mathrm{N}, 22.22$; found: $\mathrm{C}_{10} \mathrm{H}_{11} \mathrm{~N}_{3} \mathrm{O}: \mathrm{C}, 63.45 ; \mathrm{H}, 5.79 ; \mathrm{N}, 22.20$

method B- 2-propyl-4H-pyrido[2,3-d][1,3]oxazin-4-one (2) $(1 \mathrm{mmol})$ in formamide $(20 \mathrm{ml})$ was refluxed for 3 hours, the reaction mixture was cooled and poured in cold water. The obtained solid was filtered off and recrystalized from the proper solvent IR(KBr) $\mathrm{cm}^{-1}: 3215(\mathrm{NH}), 3024(\mathrm{CH}$ aromatic), 2969, 2935, 2905, 2877 (CH aliphatic), 1675 $(\mathrm{C}=\mathrm{O}), \quad 1597(\mathrm{C}=\mathrm{N}) \quad ; \mathrm{MS}: \mathrm{m} / \mathrm{z} 189\left[\mathrm{M}^{+}\right] ;{ }^{1} \mathrm{HNMR}$ (DMSO) : $\delta 8.9(\mathrm{~s}, \mathrm{H}, \mathrm{NH}), 7.2-8.2(\mathrm{~m}, 3 \mathrm{H}$, aromatic), 1.1(t, 3H, $\left.\mathrm{CH}_{3}\right), 1.9$ (sextet, $\left.2 \mathrm{H}, \mathrm{CH}_{2} \mathrm{Me}\right), 2.7\left(\mathrm{t}, 2 \mathrm{H}, \mathrm{CH}_{2}\right)$; Anal. calcd. for $\mathrm{C}_{10} \mathrm{H}_{11} \mathrm{~N}_{3} \mathrm{O}: \mathrm{C}, 63.49 ; \mathrm{H}, 5.82 ; \mathrm{N}, 22.22$; found: $\mathrm{C}_{10} \mathrm{H}_{11} \mathrm{~N}_{3} \mathrm{O}: \mathrm{C}, 63.45 ; \mathrm{H}, 5.79 ; \mathrm{N}, 22.20$

3-hydroxy-2-propylpyrido[2,3-d]pyrimidin-4(3H)-one (3c)

A solution of compound (2) (10mmol) and hydroxylamine hydrochloride in boiling ethanol $(25 \mathrm{ml})$ was refluxed for 3 hours. The reaction mixture was concentrated under reduced pressure and the residue was cooled and crystallized from the proper solvent to afford $3 \mathrm{c} . \mathrm{IR}(\mathrm{KBr}) \mathrm{cm}^{-1}$ : 3435 (OH), 3024 (CH aromatic), 2969, 2935, 2905, 2877 (CH aliphatic),1676 $(\mathrm{C}=\mathrm{O}), 1573(\mathrm{C}=\mathrm{N}) ; \mathrm{MS}: \mathrm{m} / \mathrm{z} 205$ $\left[\mathrm{M}^{+}\right] ;{ }^{1} \mathrm{HNMR}(\mathrm{DMSO}): \delta 9.7(\mathrm{~s}, \mathrm{H}, \mathrm{OH}), 7.2-8.2(\mathrm{~m}, 3 \mathrm{H}$, aromatic), 1.1(t, $3 \mathrm{H}, \mathrm{CH}_{3}$ ), 1.9 (sextet, $2 \mathrm{H}, \mathrm{CH}_{2} \mathrm{Me}$ ), 2.7 
(t,2H, $\mathrm{CH}_{2}$ ); Anal. calcd. for $\mathrm{C}_{10} \mathrm{H}_{11} \mathrm{~N}_{3} \mathrm{O}_{2}$ : C, 58.53; $\mathrm{H}$, 5.36; N, 20.48; found: C, 58.51; H, 5.32; N, 20.44 .

3-(arylideneamino)-2-propylpyrido[2,3-d]pyrimidin4(3H)-one $3 \mathrm{~d}-\mathrm{f}$

General procedure: a mixture of compound 2 (10 $\mathrm{mmol}$ ) and aromatic amines (10 $\mathrm{mmol})$ namely p-toluidine o-toluidine and, or m-toluidine in $50 \mathrm{ml}$ ethanol was stirred under condition showed in table 1 . The completion of the reaction was monitored by TLC using chloroform: methanol $(8: 2)$ as eluent and the obtained solid was filtered off and recrystalization from the ethanol solvent to give $3 \mathrm{~d}-\mathrm{f}$.

2-propyl-3-p-tolylpyrido[2,3-d]pyrimidin-4(3H)-one (3d) $\operatorname{IR}(\mathrm{KBr}) \mathrm{cm}^{-1}$ : 3024 (CH aromatic), 2969, 2935, 2905, $2877(\mathrm{CH}$ aliphatic),1676 $(\mathrm{C}=\mathrm{O}), 1573(\mathrm{C}=\mathrm{N}) ; \mathrm{MS}: \mathrm{m} / \mathrm{z}$ $205\left[\mathrm{M}^{+}\right] ;{ }^{1} \mathrm{HNMR}$ (DMSO) : $\delta 7.2-8.2(\mathrm{~m}, 7 \mathrm{H}$, aromatic), 2,9 (s, 3H, $\mathrm{CH}_{3} \mathrm{Ph}$ ), 2.5 (t, $2 \mathrm{H}, \mathrm{CH}_{2} \mathrm{CH}_{2} \mathrm{Me}$ ), 1.9 (sextet, $\left.2 \mathrm{H}, \mathrm{CH}_{2} \mathrm{Me}\right), 1.1\left(\mathrm{t}, 3 \mathrm{H}, \mathrm{CH}_{3} \mathrm{CH}_{2}\right)$; Anal. calcd. for $\mathrm{C}_{10} \mathrm{H}_{11} \mathrm{~N}_{3} \mathrm{O}_{2}$ : C, 58.53; H, 5.36; N, 20.48; found: C, 58.51; $\mathrm{H}, 5.32 ; \mathrm{N}, 20.44$.

2-propyl-3-o-tolylpyrido[2,3-d]pyrimidin-4(3H)-one (3d) IR(KBr) cm cm $^{-1} 3024$ (CH aromatic), 2969, 2935, 2905, $2877(\mathrm{CH}$ aliphatic),1676 $(\mathrm{C}=\mathrm{O}), 1573(\mathrm{C}=\mathrm{N}) ; \mathrm{MS}: \mathrm{m} / \mathrm{z}$ $205\left[\mathrm{M}^{+}\right] ;{ }^{1} \mathrm{HNMR}$ (DMSO) : $\delta 7.2-8.2(\mathrm{~m}, 7 \mathrm{H}$, aromatic), 2,9 (s, 3H, $\mathrm{CH}_{3} \mathrm{Ph}$ ), 2.5 (t, 2H, $\mathrm{CH}_{2} \mathrm{CH}_{2} \mathrm{Me}$ ), 1.9 (sextet, $\left.2 \mathrm{H}, \mathrm{CH}_{2} \mathrm{Me}\right), 1.1\left(\mathrm{t}, 3 \mathrm{H}, \mathrm{CH}_{3} \mathrm{CH}_{2}\right)$; Anal. calcd. for $\mathrm{C}_{10} \mathrm{H}_{11} \mathrm{~N}_{3} \mathrm{O}_{2}$ : C, 58.53; H, 5.36; N, 20.48; found: C, 58.51; $\mathrm{H}, 5.32 ; \mathrm{N}, 20.44$. (3d)

2-propyl-3-m-tolylpyrido[2,3-d]pyrimidin-4(3H)-one

IR(KBr) cm $\mathrm{cm}^{-1}$ : 3024 (CH aromatic), 2969, 2935, 2905, $2877(\mathrm{CH}$ aliphatic),1676 $(\mathrm{C}=\mathrm{O}), 1573(\mathrm{C}=\mathrm{N}) ; \mathrm{MS}: \mathrm{m} / \mathrm{z}$ $205\left[\mathrm{M}^{+}\right]$; ${ }^{1} \mathrm{HNMR}$ (DMSO) : $\delta 7.2-8.2$ (m, 7H, aromatic), 2,9 (s, 3H, CH $3 \mathrm{Ph}$ ), 2.5 (t, 2H, $\mathrm{CH}_{2} \mathrm{CH}_{2} \mathrm{Me}$ ), 1.9 (sextet, $\left.2 \mathrm{H}, \mathrm{CH}_{2} \mathrm{Me}\right), 1.1\left(\mathrm{t}, 3 \mathrm{H}, \mathrm{CH}_{3} \mathrm{CH}_{2}\right)$; Anal. calcd. for $\mathrm{C}_{10} \mathrm{H}_{11} \mathrm{~N}_{3} \mathrm{O}_{2}$ : C, 58.53; H, 5.36; N, 20.48; found: C, 58.51; $\mathrm{H}, 5.32 ; \mathrm{N}, 20.44$.

General procedure for preparation of 3 (Arylylideneamino) - 2- propylpyrido [2,3-d] pyrimidin $4(3 \mathrm{H})$ - ones $(4 \mathrm{a}-\mathrm{k})$

Method (A). a mixture of aromatic aldehydes (10mmol) and compound 3-amino-2-propylpyrido[2,3-d]pyrimidin$4(3 \mathrm{H})$-one (3a) $(10 \mathrm{mmol})$ in $50 \mathrm{ml}$ ethanol was reflux. The completion of the reaction was monitored by TLC using chloroform: methanol $(8: 2)$ as eluent and the obtained solid was filtered off and recrystalization from the ethanol solvent to give $4 \mathrm{a}-\mathrm{k}$.

Method (B). 3-amino-2-propylpyrido[2,3-d]pyrimidin$4(3 \mathrm{H})$-one (3a) (1 mmol, $0.204 \mathrm{~g})$ and various substituted aromatic aldehydes $(1 \mathrm{mmol})$ were added to ethanol $(1 \mathrm{~mL})$. The mixture was stirred for $5 \mathrm{~min}$ before the $0.1 \mathrm{~mol} / \mathrm{L}$ aqueous solution of iodine/potassium iodide $(10 \mathrm{~mL})$ was added to this mixture. The mixture was stirred at room temperature except for compounds $4 \mathrm{~g}$ which was refluxed for $15 \mathrm{~min}$. The completion of the reaction was monitored by TLC using chloroform: methanol (8:2) as eluent. The resultant solid was filtered and washed with sodium thiosulphate solution 5\% and hot water, respectively. The solid was pure enough but further purification can be achieved by recrystallization from ethanol.

Method (C). To a mixture of 3- amino - 2 -propylpyrido [2,3-d]pyrimidin-4(3H)-one (3a) added (1 mmol, $0.204 \mathrm{~g}$ ) and various substituted aromatic aldehydes (1 mmol), an aqueous solution of iodine/potassium iodide $(10 \mathrm{~mL}, 0.1$ $\mathrm{mol} / \mathrm{L}$ ) was added. The mixture was refluxed for the indicated time (Table 2). The precipitant was filtered and washed with sodium thiosulphate solution $5 \%$ and hot water, respectively. The solid was pure enough but further purification can be achieved by recrystallization from ethanol.

3-(benzylideneamino)-2-propylpyrido[2,3-d]pyrimidin4(3H)-one $(4 \mathrm{a})$.

$\mathrm{IR}(\mathrm{KBr}) \mathrm{cm}^{-1}: 3024$ (CH aromatic), 2969, 2935, 2905, $2877(\mathrm{CH}$ aliphatic), $1675(\mathrm{C}=\mathrm{O}), 1597(\mathrm{C}=\mathrm{N}) ; \mathrm{MS}: \mathrm{m} / \mathrm{z}$ $292\left[\mathrm{M}^{+}\right] ;{ }^{1} \mathrm{HNMR}\left(\mathrm{CHCl}_{3}\right): \delta 7.6-8.8(\mathrm{~m}, 8 \mathrm{H}$, aromatic), 6.2(s,1H, N=CH), $2.7\left(\mathrm{t}, 2 \mathrm{H}, \mathrm{CH}_{2}\right.$ pyrimidine ), 1.9 (sextet, $\left.2 \mathrm{H}, \mathrm{CH}_{2} \mathrm{Me}\right), \quad 1.1\left(\mathrm{t}, 3 \mathrm{H}, \mathrm{CH}_{3}\right)$. Anal. calcd. for $\mathrm{C}_{17} \mathrm{H}_{16} \mathrm{~N}_{4} \mathrm{O}: \mathrm{C}, 69.86$; H, 5.47; N, 19.17; found: C, 69.82; $\mathrm{H}, 5.44 ; \mathrm{N}, 19.15$.

3-(p-tolylideneamino)-2-propylpyrido[2,3-d]pyrimidin4(3H)-one $(4 \mathrm{~b})$.

IR(KBr) cm $\mathrm{cm}^{-1}: 3024$ (CH aromatic), 2955, 2945, 2915, 2867 (CH aliphatic), $1673(\mathrm{C}=\mathrm{O}), 1598(\mathrm{C}=\mathrm{N}) ; \mathrm{MS}: \mathrm{m} / \mathrm{z}$ $292\left[\mathrm{M}^{+}\right] ;{ }^{1} \mathrm{HNMR}\left(\mathrm{CHCl}_{3}\right): \delta 7.6-8.8(\mathrm{~m}, 7 \mathrm{H}$, aromatic), 6.1(s,1H, N=CH), $2.35\left(\mathrm{~s}, 3 \mathrm{H}, \mathrm{CH}_{3}\right.$ phenyl), $2.7(\mathrm{t}, 2 \mathrm{H}$, $\mathrm{CH}_{2}$ pyrimidine), 1.9 (sextet,2H, $\left.\mathrm{CH}_{2} \mathrm{Me}\right), 1.1\left(\mathrm{t}, 3 \mathrm{H}, \mathrm{CH}_{3}\right.$ ). Anal. calcd. for $\mathrm{C}_{18} \mathrm{H}_{18} \mathrm{~N}_{4} \mathrm{O}: \mathrm{C}, 70.12 ; \mathrm{H}, 5.84 ; \mathrm{N}, 18.18$; found: C, 70.16; H, 5.81; N, 18.19 .

3-(4-chlorobenzylideneamino)-2-propylpyrido[2,3d]pyrimidin-4(3H)-one(4c).

$\mathrm{IR}(\mathrm{KBr}) \mathrm{cm}^{-1}: 3030$ (CH aromatic), 2975, 2939, 2911, $2876(\mathrm{CH}$ aliphatic),1671 (C=O ), 1594(C=N) ; MS: m/z $326.5\left[\mathrm{M}^{+}\right] ;{ }^{1} \mathrm{HNMR}\left(\mathrm{CHCl}_{3}\right): \delta 7.6-8.8(\mathrm{~m}, 7 \mathrm{H}$, aromatic), $6.4(\mathrm{~s}, 1 \mathrm{H}, \mathrm{N}=\mathrm{CH}), 2.7\left(\mathrm{t}, 2 \mathrm{H}, \mathrm{CH}_{2}\right.$ pyrimidine $), 1.9$ (sextet,2H, $\mathrm{CH}_{2} \mathrm{Me}$ ), 1.1(t, 3H, $\left.\mathrm{CH}_{3}\right)$. Anal. calcd. for $\mathrm{C}_{17} \mathrm{H}_{15} \mathrm{~N}_{4} \mathrm{OCl}$ : C, 62.48; H, 4.59; N, 17.15; found: C, 69.82; $\mathrm{H}, 5.44 ; \mathrm{N}, 19.15$.

3-(4-nitrobenzylideneamino)-2-propylpyrido[2,3d]pyrimidin-4(3H)-one (4d).

$\mathrm{IR}(\mathrm{KBr}) \mathrm{cm}^{-1}: 3022$ (CH aromatic), 2968, 2945, 2909, $2871(\mathrm{CH}$ aliphatic),1677 (C=O ), 1599(C=N) ; MS: m/z $337\left[\mathrm{M}^{+}\right] ;{ }^{1} \mathrm{HNMR}\left(\mathrm{CHCl}_{3}\right): \delta 7.6-8.8(\mathrm{~m}, 7 \mathrm{H}$, aromatic), $6.4(\mathrm{~s}, 1 \mathrm{H}, \mathrm{N}=\mathrm{CH}) 2.7\left(\mathrm{t}, 2 \mathrm{H}, \mathrm{CH}_{2}\right.$ pyrimidine ), 1.9 (sextet, $\left.2 \mathrm{H}, \mathrm{CH}_{2} \mathrm{Me}\right), \quad 1.1\left(\mathrm{t}, 3 \mathrm{H}, \mathrm{CH}_{3}\right)$. Anal. calcd. for $\mathrm{C}_{17} \mathrm{H}_{15} \mathrm{~N}_{5} \mathrm{O}_{3}$ : C, 60.53; H, 4.45; N, 20.77; found: C, 60.52; $\mathrm{H}, 4.41 ; \mathrm{N}, 20.74$

3-(3-nitrobenzylideneamino)-2-propylpyrido[2,3d]pyrimidin-4(3H)-one (4e).

$\mathrm{IR}(\mathrm{KBr}) \mathrm{cm}^{-1}$ : 3022 (CH aromatic), 2968, 2945, 2909, $2871(\mathrm{CH}$ aliphatic),1677 (C=O ), 1599(C=N) ; MS: m/z $337\left[\mathrm{M}^{+}\right]$; ${ }^{1} \mathrm{HNMR}\left(\mathrm{CHCl}_{3}\right): \delta 7.6-8.8(\mathrm{~m}, 7 \mathrm{H}$, aromatic), $6.2(\mathrm{~s}, 1 \mathrm{H}, \mathrm{N}=\mathrm{CH}) 2.7\left(\mathrm{t}, 2 \mathrm{H}, \mathrm{CH}_{2}\right.$ pyrimidine ), 1.9 (sextet, $\left.2 \mathrm{H}, \mathrm{CH}_{2} \mathrm{Me}\right), \quad 1.1\left(\mathrm{t}, 3 \mathrm{H}, \mathrm{CH}_{3}\right)$. Anal. calcd. for $\mathrm{C}_{17} \mathrm{H}_{15} \mathrm{~N}_{5} \mathrm{O}_{3}$ : C, 60.53; H, 4.45; N, 20.77; found: $\mathrm{C}, 60.52$; $\mathrm{H}, 4.41 ; \mathrm{N}, 20.74$ 
3-(3-methoxybenzylideneamino)-2-propylpyrido[2,3d]pyrimidin-4(3H)-one (4f).

$\mathrm{IR}(\mathrm{KBr}) \mathrm{cm}^{-1}: 3022$ (CH aromatic), 2979, 2945, 2915, $2870(\mathrm{CH}$ aliphatic $), 1673(\mathrm{C}=\mathrm{O}), 1596(\mathrm{C}=\mathrm{N}) ; \mathrm{MS}: \mathrm{m} / \mathrm{z}$ $322\left[\mathrm{M}^{+}\right] ;{ }^{1} \mathrm{HNMR}\left(\mathrm{CHCl}_{3}\right): \delta 7.6-8.8(\mathrm{~m}, 7 \mathrm{H}$, aromatic), $6.4(\mathrm{~s}, 1 \mathrm{H}, \mathrm{N}=\mathrm{CH}), 3.75\left(\mathrm{~s}, 3 \mathrm{H}, \mathrm{O} \mathrm{CH}_{3}\right), 2.7(\mathrm{t}, 2 \mathrm{H}$, $\mathrm{CH}_{2}$ pyrimidine), 1.9 (sextet, $2 \mathrm{H}, \mathrm{CH}_{2} \mathrm{Me}$ ), $1.1\left(\mathrm{t}, 3 \mathrm{H}, \mathrm{CH}_{3}\right.$ ). Anal. calcd. for $\mathrm{C}_{18} \mathrm{H}_{18} \mathrm{~N}_{4} \mathrm{O}_{2}$ : C, 67.08; H, 5.59; N, 17.39; found: $\mathrm{C}, 67.02 ; \mathrm{H}, 5.54 ; \mathrm{N}, 17.37$.

3-(4-methoxybenzylideneamino)-2-propylpyrido[2,3d]pyrimidin-4(3H)-one (4g).

$\operatorname{IR}(\mathrm{KBr}) \mathrm{cm}^{-1}: 3022$ (CH aromatic), 2979, 2945, 2915, $2870(\mathrm{CH}$ aliphatic), $1673(\mathrm{C}=\mathrm{O}), 1596(\mathrm{C}=\mathrm{N}) ; \mathrm{MS}: \mathrm{m} / \mathrm{z}$ $322\left[\mathrm{M}^{+}\right] ;{ }^{1} \mathrm{HNMR}\left(\mathrm{CHCl}_{3}\right): \delta 7.6-8.8(\mathrm{~m}, 7 \mathrm{H}$, aromatic), $6.4(\mathrm{~s}, 1 \mathrm{H}, \mathrm{N}=\mathrm{CH}), 3.75\left(\mathrm{~s}, 3 \mathrm{H}, \mathrm{O} \mathrm{CH}_{3}\right), 2.7(\mathrm{t}, 2 \mathrm{H}$, $\mathrm{CH}_{2}$ pyrimidine), 1.9 (sextet, $2 \mathrm{H}, \mathrm{CH}_{2} \mathrm{Me}$ ), $1.1\left(\mathrm{t}, 3 \mathrm{H}, \mathrm{CH}_{3}\right.$ ). Anal. calcd. for $\mathrm{C}_{18} \mathrm{H}_{18} \mathrm{~N}_{4} \mathrm{O}_{2}$ : C, 67.08; H, 5.59; N, 17.39; found: $\mathrm{C}, 67.02 ; \mathrm{H}, 5.54 ; \mathrm{N}, 17.37$.

3-(2-methoxybenzylideneamino)-2-propylpyrido[2,3d]pyrimidin-4(3H)-one (4h).

$\mathrm{IR}(\mathrm{KBr}) \mathrm{cm}^{-1}: 3022$ (CH aromatic), 2979, 2945, 2915, $2870(\mathrm{CH}$ aliphatic $), 1673(\mathrm{C}=\mathrm{O}), 1596(\mathrm{C}=\mathrm{N}) ; \mathrm{MS}: \mathrm{m} / \mathrm{z}$ $322\left[\mathrm{M}^{+}\right] ;{ }^{1} \mathrm{HNMR}\left(\mathrm{CHCl}_{3}\right): \delta 7.6-8.8(\mathrm{~m}, 7 \mathrm{H}$, aromatic), $6.4(\mathrm{~s}, 1 \mathrm{H}, \mathrm{N}=\mathrm{CH}), 3.75\left(\mathrm{~s}, 3 \mathrm{H}, \mathrm{O} \mathrm{CH} \mathrm{CH}_{3}\right), 2.7(\mathrm{t}, 2 \mathrm{H}$, $\mathrm{CH}_{2}$ pyrimidine), 1.9 (sextet, $\left.2 \mathrm{H}, \mathrm{CH}_{2} \mathrm{Me}\right), 1.1\left(\mathrm{t}, 3 \mathrm{H}, \mathrm{CH}_{3}\right.$ ). Anal. calcd. for $\mathrm{C}_{18} \mathrm{H}_{18} \mathrm{~N}_{4} \mathrm{O}_{2}$ : C, 67.08; H, 5.59; N, 17.39; found: $\mathrm{C}, 67.10 ; \mathrm{H}, 5.55 ; \mathrm{N}, 17.36$

3-(4-hydroxybenzylideneamino)-2-propylpyrido[2,3d]pyrimidin-4(3H)-one (4i).

$\mathrm{IR}(\mathrm{KBr}) \mathrm{cm}^{-1}: 3024$ (CH aromatic), 2969, 2935, 2905, $2877(\mathrm{CH}$ aliphatic $), 1675(\mathrm{C}=\mathrm{O}), 1597(\mathrm{C}=\mathrm{N}) ; \mathrm{MS}: \mathrm{m} / \mathrm{z}$ $310\left[\mathrm{M}^{+}\right] ;{ }^{1} \mathrm{HNMR}\left(\mathrm{CHCl}_{3}\right): \delta 7.6-8.8(\mathrm{~m}, 7 \mathrm{H}$, aromatic), $6.4(\mathrm{~s}, 1 \mathrm{H}, \quad \mathrm{N}=\mathrm{CH}), \quad 5.25(\mathrm{~s}, \mathrm{H}, \mathrm{OH}), 2.7(\mathrm{t}, 2 \mathrm{H}$, $\mathrm{CH}_{2}$ pyrimidine), 1.9 (sextet, $2 \mathrm{H}, \mathrm{CH}_{2} \mathrm{Me}$ ), $1.1\left(\mathrm{t}, 3 \mathrm{H}, \mathrm{CH}_{3}\right.$ ). Anal. calcd. for $\mathrm{C}_{17} \mathrm{H}_{16} \mathrm{~N}_{4} \mathrm{O}_{2}$ : C, 65.80; H, 5.16; N, 18.06; found: C, 65.77; H, 5.12; N, 18.03 .

3-(2-hydroxybenzylideneamino)-2-propylpyrido[2,3d]pyrimidin-4(3H)-one $(4 \mathrm{j})$.

$\operatorname{IR}(\mathrm{KBr}) \mathrm{cm}^{-1}: 3024$ (CH aromatic), 2969, 2935, 2905, $2877(\mathrm{CH}$ aliphatic), $1675(\mathrm{C}=\mathrm{O}), 1597(\mathrm{C}=\mathrm{N}) ; \mathrm{MS}: \mathrm{m} / \mathrm{z}$ $310\left[\mathrm{M}^{+}\right] ;{ }^{1} \mathrm{HNMR}\left(\mathrm{CHCl}_{3}\right): \delta 7.6-8.8(\mathrm{~m}, 7 \mathrm{H}$, aromatic), $6.3(\mathrm{~s}, 1 \mathrm{H}, \quad \mathrm{N}=\mathrm{CH}), \quad 5.25 \quad(\mathrm{~s}, \quad \mathrm{H}, \mathrm{OH}), 2.7 \quad(\mathrm{t}, 2 \mathrm{H}$, $\mathrm{CH}_{2}$ pyrimidine), 1.9 (sextet, $2 \mathrm{H}, \mathrm{CH}_{2} \mathrm{Me}$ ), $1.1\left(\mathrm{t}, 3 \mathrm{H}, \mathrm{CH}_{3}\right.$ ). Anal. calcd. for $\mathrm{C}_{17} \mathrm{H}_{16} \mathrm{~N}_{4} \mathrm{O}_{2}$ : C, 65.80; H, 5.16; N, 18.06; found: C, 65.77; H, 5.12; N, 18.03.

3-(3-hydroxybenzylideneamino)-2-propylpyrido[2,3d]pyrimidin-4(3H)-one (4k).

$\mathrm{IR}(\mathrm{KBr}) \mathrm{cm}^{-1}: 3024$ (CH aromatic), 2969, 2935, 2905, $2877(\mathrm{CH}$ aliphatic),1675 $(\mathrm{C}=\mathrm{O}), 1597(\mathrm{C}=\mathrm{N}) ; \mathrm{MS}: \mathrm{m} / \mathrm{z}$ $310\left[\mathrm{M}^{+}\right] ;{ }^{1} \mathrm{HNMR}\left(\mathrm{CHCl}_{3}\right): \delta 7.6-8.8(\mathrm{~m}, 7 \mathrm{H}$, aromatic), $6.5(\mathrm{~s}, 1 \mathrm{H}, \quad \mathrm{N}=\mathrm{CH}) \quad 5.25 \quad(\mathrm{~s}, \mathrm{H} \quad \mathrm{OH}), 2.7(\mathrm{t}, 2 \mathrm{H}$, $\mathrm{CH}_{2}$ pyrimidine), 1.9 (sextet, $\left.2 \mathrm{H}, \mathrm{CH}_{2} \mathrm{Me}\right), 1.1\left(\mathrm{t}, 3 \mathrm{H}, \mathrm{CH}_{3}\right.$ ). Anal. calcd. for $\mathrm{C}_{17} \mathrm{H}_{16} \mathrm{~N}_{4} \mathrm{O}_{2}$ : C, 65.80; H, 5.16; N, 18.06; found: C, 65.77; H, 5.12; N, 18.03.

\section{Acknowledgements}

The authors are thankful to Basic \& Health Science Research Center, Deanship of research Majmaah university for the financial support.

\section{References}

[1] S.N. VanderWel, P.J. Harvey, D.J. McNamara, J.T. Repine, P.R. Keller, J. Quin III, R.J. Booth, W.L. Elliott, E.M. Dobrusin, D.W. Fry, P.L. Toogood, J. Med. Chem 48 (2005) $2371 \mathrm{e} 2378$.

[2] P.L. Toogood, P.J. Harvey, J.T. Repine, D.J. Sheehan, S.N. VanderWel, H. Zhou, P.R. Keller, D.J. McNamara, D. Sherry, T. Zhu, J. Brodfuehrer, C. Choi, M.R. Barvian, D.W. Fry, J. Med. Chem 48 (2005) 2388e2406.

[3] B.D. Palmer, J.B. Smaill, G.W. Rewcastle, E.M. Dobrusin, A. Kraker, C.W. Moore, R.W. Steinkampf, W.A. Denny, Bioorg. Med.

[4] K. Malagu, H. Duggan, K. Menear, M. Hummersone, S. Gomez, C. Bailey, P. Edwards, J. Drzewiecki, F. Leroux, M.J. Quesada, G. Hermann, S. Maine, C. Molyneaux, A. Le Gall, J. Pullen, I. Hickson, L. Smith, S. Maguire, N. Martin, G. Smith, M. Pass, Bioorg. Med. Chem. Lett. 19 (2009) $5950 \mathrm{e} 5953$.

[5] X.L. Zhao, Y.F. Zhao, S.C. Guo, H.S. Song, D. Wang, P. Gong, Molecules 12 (2007) 1136e1146.

[6] H.N. Hafez, A.B.A. El-Gazzar, Bioorg. Med. Chem. Lett. 19 (2009) 4143e4147.

[7] A.S. Shawali, S.M. Sherif, M.A.A. Darwish, M.M. Elmerzabani, Arch. Pharm. Res. 33 (2010) 55e60.

[8] (a) Masuda, Satoru et al. Jpn.Kokai. Tokkyo Koho Jp 03, 106, 880. C.A.15: 183361d. (b) Tamura et al. Jpn. Kokai. Tokkyo Koho Jp 61, 249, 983, C.A. 106: 213979.

[9] J. Ram, D.A. Vanden Berghe, A.J.J. Vlietinck, Heterocycl. Chem. 28 (1988) 217.

[10] J.R. Piper, G.S. Mc Calab, J.A. Montgomery, R.L. Kishiuk, Y. Gamount, F.M. Sirotnak, J. Med. Chem. 29 (1986) $1080 \mathrm{e} 1087$.

[11] R.K. Robins, G.H.J. Hitchings, Am. Chem. Soc. 80 (1958) 3449 .

[12] M.F. Hasan, A.M. Madkour, I. Saleem, J.M.A. Rahman, E.A.Z. Mohammed, Heterocycles 38 (1994) 57.

[13] John Adams Lowe,Chem. Abstr. 112 (1990) 21008 Austrian At. 388378 (1989) 378.

[14] A. Gangjee, O.O. Adair, S.F. Queener, J. Med. Chem. 46 (2003) 5074e5082. H. Iwamura, S. Murakami, K. Koshimizu, S. Matsabura, J. Med. Chem. 28 (1985)577e583. 